\title{
Adipocyte/macrophage fatty acid-binding proteins contribute to metabolic deterioration through actions in both macrophages and adipocytes in mice
}

\author{
Masato Furuhashi, Raquel Fucho, Cem Z. Görgün, Gürol Tuncman, \\ Haiming Cao, and Gökhan S. Hotamisligil
}

Department of Genetics and Complex Diseases, Harvard School of Public Health, Boston, Massachusetts, USA.

\begin{abstract}
Adipose tissue inflammation is a characteristic of obesity. However, the mechanisms that regulate this inflammatory response and link adipose inflammation to systemic metabolic consequences are not fully understood. In this study, we have taken advantage of the highly restricted coexpression of adipocyte/macrophage fatty acid-binding proteins (FABPs) aP2 (FABP4) and mal1 (FABP5) to examine the contribution of these lipid chaperones in macrophages and adipocytes to local and systemic inflammation and metabolic homeostasis in mice. Deletion of FABPs in adipocytes resulted in reduced expression of inflammatory cytokines in macrophages, whereas the same deletion in macrophages led to enhanced insulin signaling and glucose uptake in adipocytes. Using radiation chimerism through bone marrow transplantation, we generated mice with FABP deficiency in bone marrow and stroma-derived elements in vivo and studied the impact of each cellular target on local and systemic insulin action and glucose metabolism in dietary obesity. The results of these experiments indicated that neither macrophages nor adipocytes individually could account for the total impact of FABPs on systemic metabolism and suggest that interactions between these 2 cell types, particularly in adipose tissue, are critical for the inflammatory basis of metabolic deterioration.
\end{abstract}

\section{Introduction}

Interactions between metabolic and inflammatory response systems play a significant role in the pathogenesis of a cluster of chronic metabolic diseases, including type 2 diabetes, fatty liver disease, and atherosclerosis (1). Adipose tissue represents a criti$\mathrm{cal}$ and predominant site for the interactions between metabolic and inflammatory responses, and adipocytes harbor the capacity to produce numerous inflammatory mediators, especially under conditions of stress, such as during obesity (1). In addition to these cell-autonomous responses, recent studies have also demonstrated the presence of immune cells within adipose tissue during obesity, raising the possibility of contribution of these cells to the inflammatory changes as well as metabolic deterioration. For example, macrophage infiltration in adipose tissue has recently been described in both mice and humans, especially in the later stages of obesity $(2,3)$. It has been suggested that expanding adipocytes or neighboring preadipocytes might produce signals leading to macrophage recruitment (4). Alternatively, death of adipocytes at late stages of obesity has also been proposed as a mechanism of macrophage infiltration into the adipose tissue (5). In fact, this is a very attractive hypothesis, as much of the macrophage presence in adipose tissue is in a scattered pattern and found around the dead adipocytes in obesity. These observations have raised the possibility that macrophages themselves might be a critical regulator

Nonstandard abbreviations used: BMT, bone marrow transplantation/bone marrow-transplanted; FABP, fatty acid-binding protein; MCP-1, monocyte chemoattractant protein-1; SV, stromal-vascular.

Conflict of interest: The authors have declared that no conflict of interest exists. Citation for this article: J. Clin. Invest. 118:2640-2650 (2008). doi:10.1172/JCI34750. of metabolism as a result of their inflammatory capacity, perhaps independent of stromal counterparts, especially adipocytes. Several studies have identified contributions to metabolic regulation of pathways that act in the macrophage $(6,7)$. Similarly, specific and isolated deletion of target genes in the myeloid lineage has also produced support for the hypothesis that these cells impact systemic metabolic homeostasis $(8,9)$. On the other hand, there remain challenges in understanding the interactions within adipose tissue, since experimental paradigms to restrict targeted gene expression to macrophages in vivo are limited, and most targeted genes act on other key metabolic sites that could impact systemic glucose and lipid homeostasis. Hence, our understanding of the contribution of macrophages or specific pathways in macrophages per se on local or systemic insulin sensitivity and glucose metabolism remains incomplete, particularly in adipose tissue.

In this study, we have attempted to take advantage of the highly cell type-restricted coexpression of adipocyte/macrophage lipid chaperones to address the role of interactions between adipose tissue macrophages and adipocytes in dietary obesity and how these interactions synergize to regulate metabolic homeostasis in mice. The lipid chaperone proteins, also known as fatty acid-binding proteins (FABPs), are a group of molecules that coordinate inflammatory and metabolic responses in adipocytes and macrophages (10). These proteins are a family of 14 - to $15-\mathrm{kDa}$ proteins that bind with high affinity to hydrophobic ligands such as saturated and unsaturated long-chain fatty acids (10). Two isoforms of FABPs, aP2 (FABP4) and mal1 (FABP5), are highly similar in sequence and structure to each other and are the only isoforms coexpressed in adipocytes and macrophages (10-12). In our previous studies, we have demonstrated the exclusive presence of these 
A

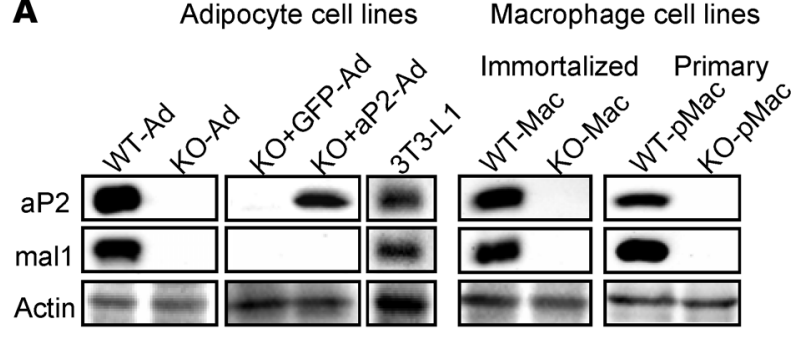

B

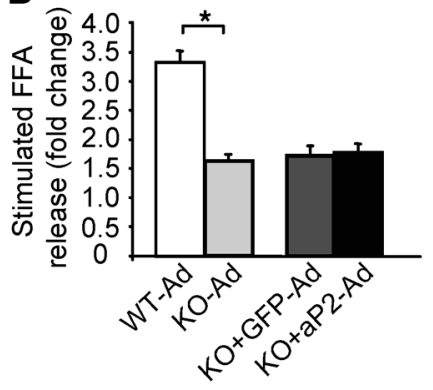

C

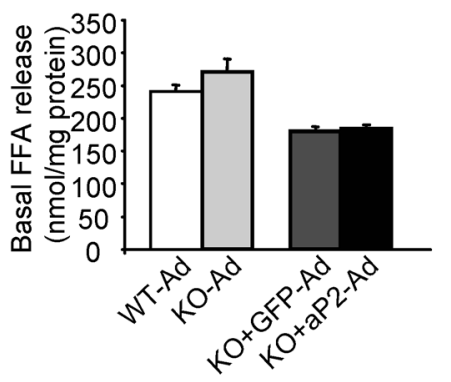

D

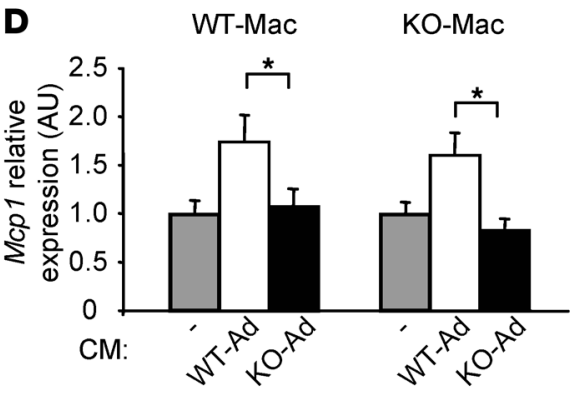

E

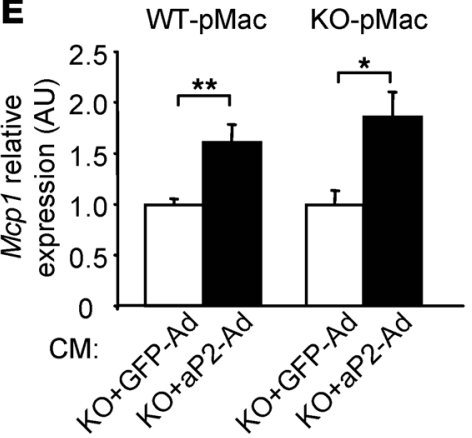

$\mathbf{F}$

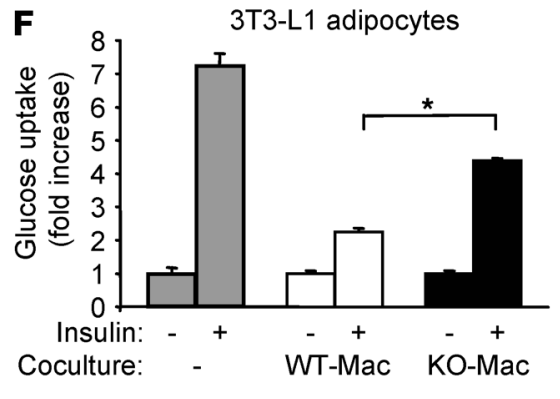

H KO+GFP-Ad KO+aP2-Ad

G

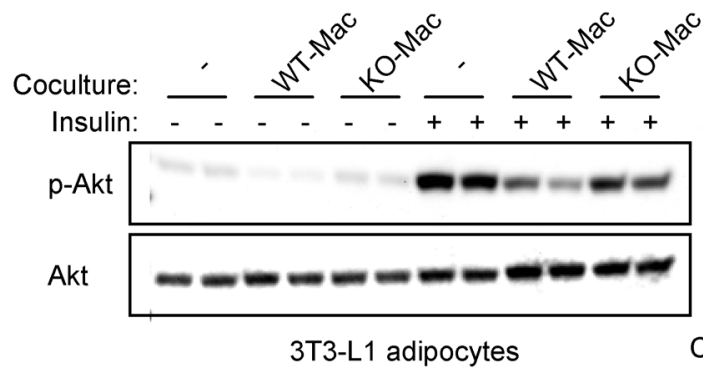

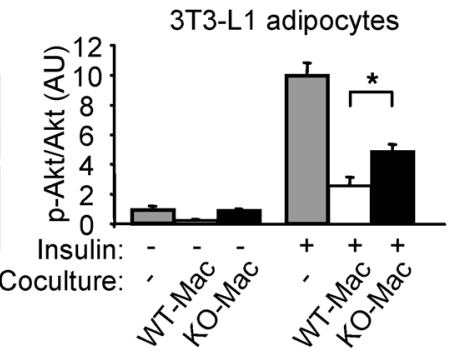

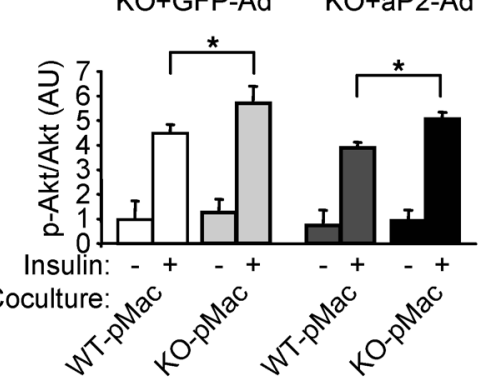

Figure 1

Coculture experiments. (A) Protein expression of aP2 and mal1 in adipocyte cell lines (WT-Ad, KO-Ad, KO+aP2-Ad, KO+GFP-Ad, and 3T3-L1), mouse macrophage cell lines (WT-Mac and KO-Mac), and thioglycollate-elicited primary macrophages from Ap2 $2^{+/} \mathrm{Mal} \mathrm{T}^{+/+}$(WT-pMac) and $\mathrm{Ap} 2^{-/-M a / 1^{-/-}}$ (KO-pMac) mice. (B) Dibutyryl cAMP-stimulated lipolysis (4-hour stimulated FFA release) in adipocyte cell lines. (C) Basal FFA release into the conditioned medium (CM) in adipocytes examined under the same conditions of coculture experiments for 16 hours. (D) Expression of $M c p 1$ in macrophages, WT-Mac or KO-Mac, incubated with conditioned medium from WT-Ad or KO-Ad adipocytes. Data were normalized to those in untreated macrophages. (E) Expression of Mcp1 in primary macrophages, WT-pMac or KO-pMac, incubated with conditioned medium from KO+GFP-Ad (FABP-deficient) or KO+aP2-Ad (FABP-reconstituted) adipocytes. Data were normalized to those in macrophages incubated with the conditioned medium from KO+GFP-Ad adipocytes. (F) Insulin-stimulated glucose uptake in 3T3-L1 adipocytes incubated in contact with immortalized macrophages, WT-Mac or KO-Mac. (G) Insulin-stimulated phosphorylation of Akt in 3T3-L1 adipocytes incubated in contact with immortalized macrophages. The graph on the right shows the quantification. $(\mathbf{H})$ Insulin-stimulated phosphorylation of Akt in adipocyte cell lines, KO+GFP-Ad and KO+aP2-Ad, incubated in contact with primary macrophages, WT-pMac or KO-pMac. Data are shown as mean \pm SEM. ${ }^{*} P<0.05,{ }^{*} P<0.01$.

FABPs in macrophages among all of the bone marrow-derived cells, either in the resting or activated stage $(12,13)$. Furthermore, studies in $\mathrm{Ap} 2^{-{ }^{-}}, \mathrm{Mal1}^{--}$, and $\mathrm{Ap} \mathrm{2}^{-/-} \mathrm{Mal1}^{-{ }^{--}}$mice have shown that these lipid chaperones play a significant role in several aspects of the metabolic syndrome, including insulin resistance, type 2 diabetes, and atherosclerosis (12-19), through their ability to act at the interface of metabolic and inflammatory pathways in the cells that they target. Most importantly, recent reports have provided compelling evidence that these actions are also relevant to human disease and raised the possibility of utilizing FABPs in general, especially aP2, as potential drug targets to treat metabolic diseases $(20,21)$. We have recently provided a critical proof of principle that aP2 can be successfully targeted by an orally active small molecule inhibitor to generate a profile reminiscent of genetic deficiency in vitro and in vivo (22). Hence, adipocyte/ macrophage lipid chaperones may offer a unique opportunity to examine the interactions between adipose tissue macrophages and adipocytes in a model highly relevant to human disease. In addition to understanding macrophage-adipocyte interactions, therapeutic considerations also make it critical to dissect the cellular targets of FABPs as they relate to metabolic regulation. Most critical is the fact that adipocytes express very high levels of aP2 compared with macrophages (approximately 10,000-fold greater) (23). Hence, if macrophages or nonstromal elements contribute 
A

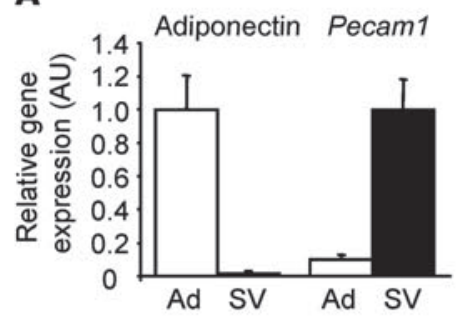

D
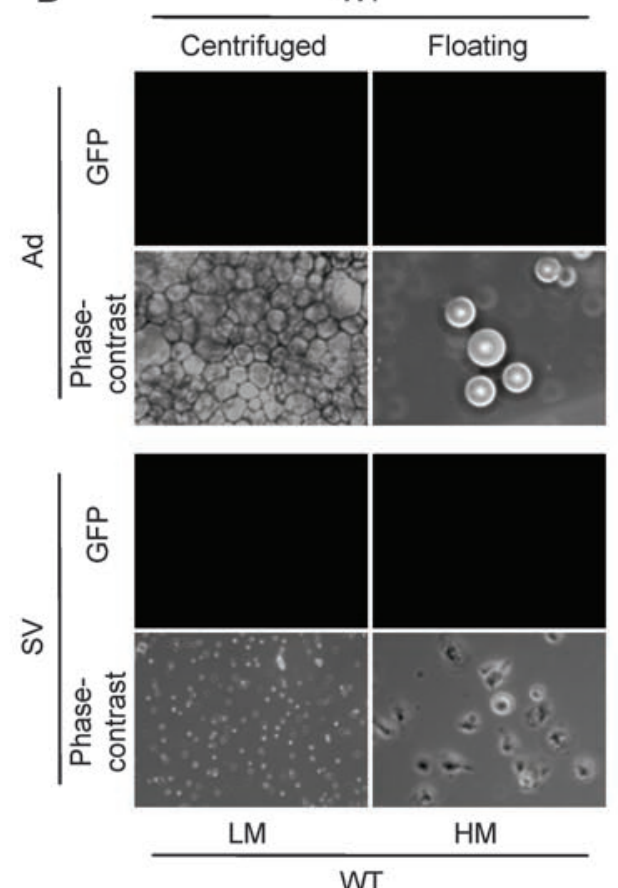

B

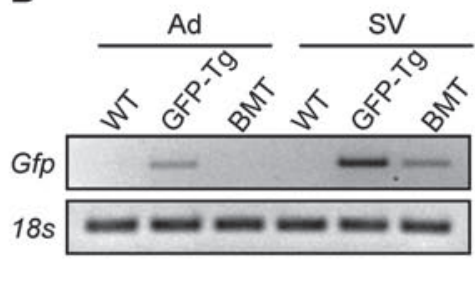

C

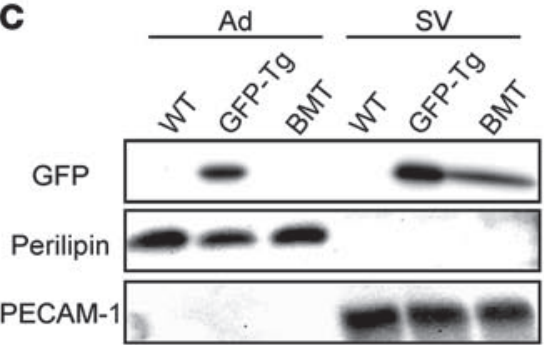

BMT (GFP-Tg $\rightarrow$ WT)
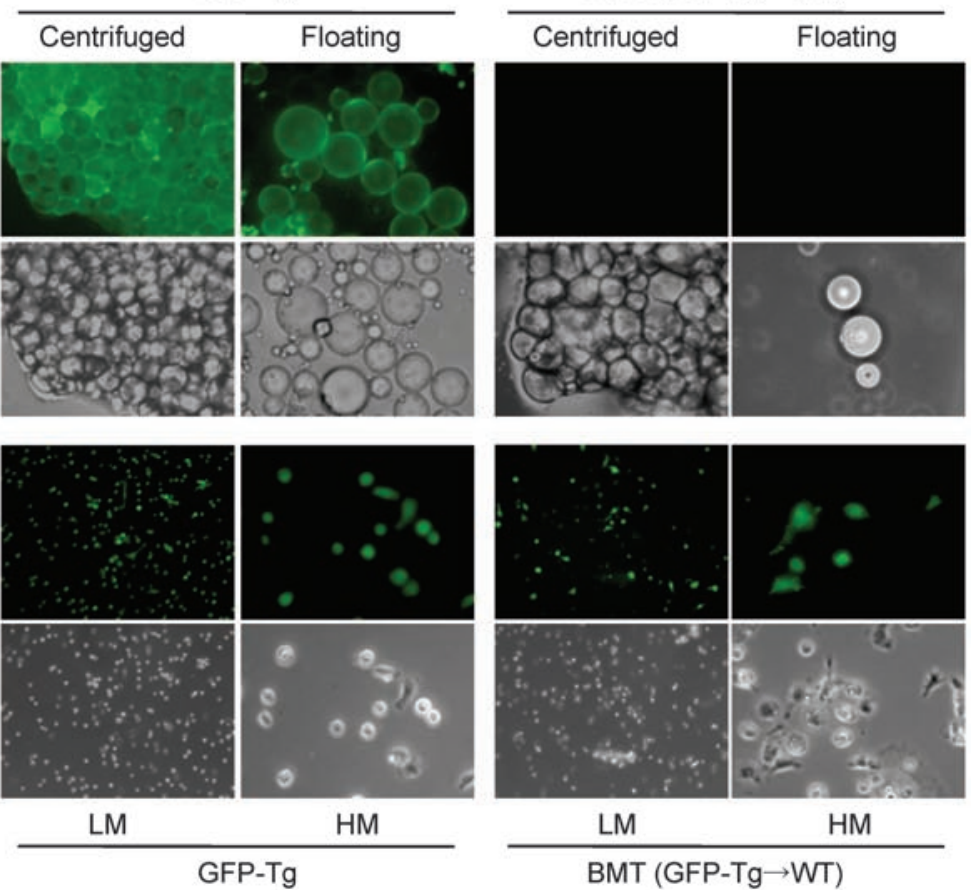

Figure 2

Adipocyte and SV fractions in BMT (GFP-Tg $\rightarrow$ WT) mice. GFP-labeled donor cells were used to track the destiny of bone marrow-derived cells. (A) Confirmation of the adipocyte (Ad) and SV fractions from WT, GFP-transgenic (GFP-Tg), and BMT (GFP-Tg $\rightarrow$ WT) mice by differential expression of adiponectin as an adipocyte and Pecam1 as a non-adipocyte SV marker. Data are shown as mean \pm SEM. (B) Detection of Gfp mRNA by PCR in the same fractions shown in A. (C) Detection of GFP protein by Western blotting in the same fractions. Perilipin and PECAM-1 were controls of the Ad and SV fractions, respectively. (D) Fluorescence microscopic analysis of the adipose tissue fractions. In the adipocyte fraction, images were taken in both the centrifuged fat pad after digestion or in floating fat cells in DMEM with 10\% cosmic calf serum (magnification, $\times 200)$. In the SV fraction, cells were observed at both the low and high magnifications (LM, $\times 100$; and HM, $\times 400)$.

to FABP-regulated insulin sensitivity, chemical inhibition of this pathway might be more realistic, as it would not require complete targeting of $\mathrm{aP} 2$ in adipocytes for efficacy.

Here, we examined the interactions between adipocytes and macrophages and the impact of lipid chaperones on these functional interactions using macrophage and adipocyte cell lines and whole animals. These studies demonstrated that FABP action in either adipocytes or macrophages is critical for the inflammatory and metabolic responses of these cells in vitro and that both sites could impact systemic insulin sensitivity and glucose metabolism in vivo.

\section{Results}

Regulation of functional interactions between adipocytes and macrophages by FABPs. To test the specific role of FABPs in both adipocytes and macrophages and the interactions between these cells, we developed cellular coculture systems using several adipocyte and macrophage cell lines. Preadipocyte cell lines from WT and FABP-deficient mice (WT-Ad and KO-Ad, respectively) were developed in our laboratory as previously described (22). In addition, FABP-deficient preadipocytes were reconstituted with exogenous aP2 (KO+aP2-Ad) or control empty vector (KO+GFP-Ad) to establish controls within the identical cellular background. These preadipocyte cell lines fully differentiate into adipocytes and behave similarly to commonly used 3T3-L1 or 3T3-F442A adipocytes (22). In addition to these adipocytes, we established macrophage cell lines from WT and FABP-deficient mice (WT-Mac and KO-Mac, respectively). To complement this system, we also used primary macrophages from WT and FABP-deficient mice elicited by an intraperitoneal injection of thioglycollate (WT-pMac and KO-pMac, respectively). In all of these cellular systems, we examined FABP expression status. As shown in Figure 1A, expression of FABPs in the cells was confirmed by Western blot analysis with isoform-specific antibod- 
A

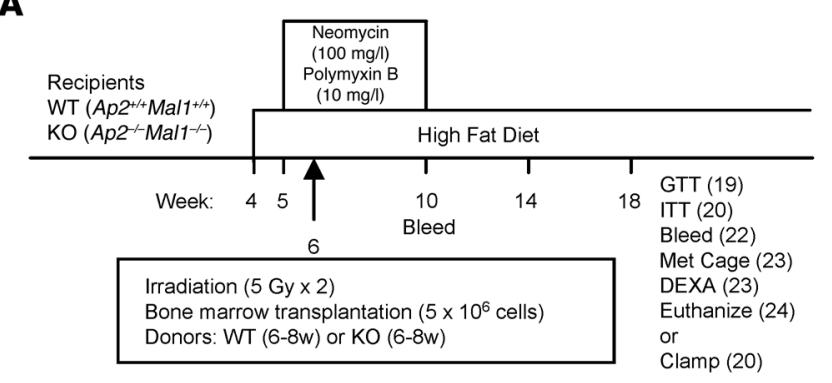

C
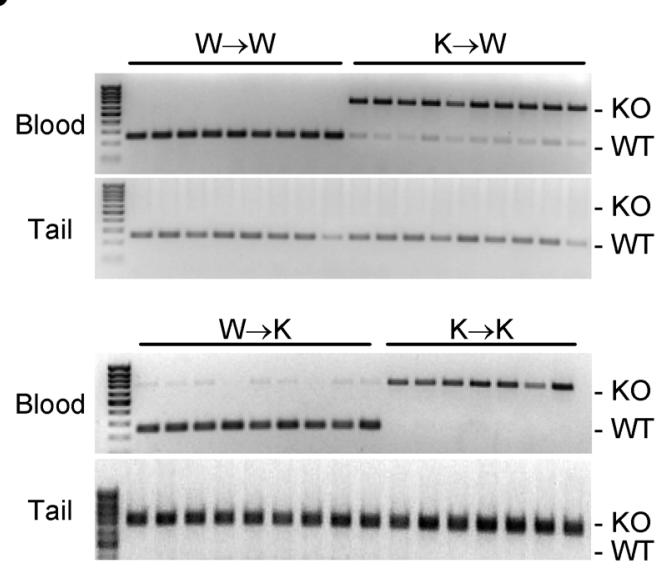

B

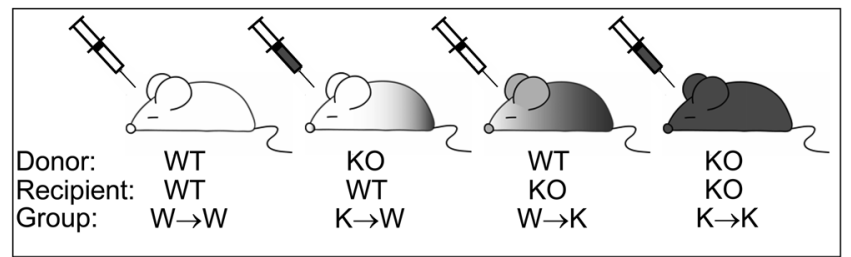

Blood
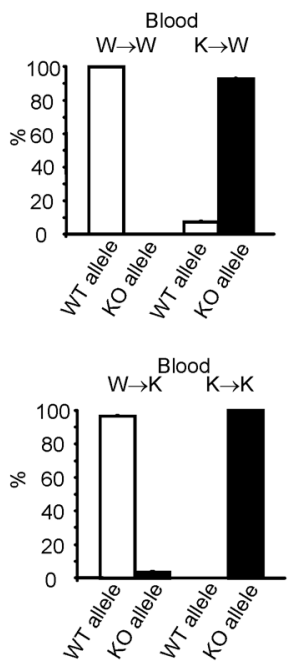

D

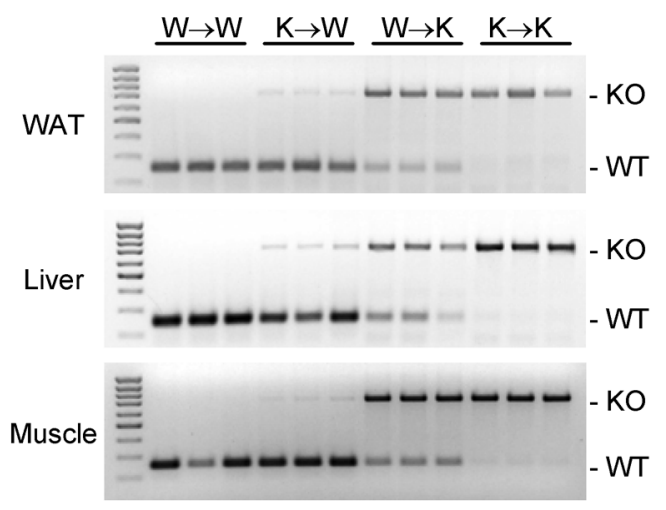

\section{Figure 3}

BMT experiments in FABP-deficient mice. (A) Experimental design of the BMT studies using WT $\left(A p 2^{+/+} M a / 1^{+/+}\right)$and FABP-deficient $\left(A p 2^{-/-M a l 1^{-/-}}\right)$ mice. The numbers in parentheses indicate the timing (week) of performed items. (B) Description and nomenclature for the groups of BMT mice. (C) Genotyping using DNA samples from blood and tail in BMT mice. The graphs on the right show the quantification for the percentages of WT and $\mathrm{KO}$ alleles in blood samples. Data are shown as mean \pm SEM. (D) Genotyping using DNA samples from white adipose tissue (WAT), liver, and skeletal muscle (soleus) in BMT mice for the donor and recipient alleles. GTT, glucose tolerance test; ITT, insulin tolerance test; Met Cage, metabolic cage study; DEXA, dual energy x-ray absorptiometry; Clamp, hyperinsulinemic-euglycemic clamp study.

ies. We also examined lipolysis in adipocyte cell lines used in this study. Similar to what was previously observed $(24,25)$, there was a significant reduction in stimulated lipolysis in KO-Ad compared with WT-Ad cell lines (Figure 1B), but levels of basal release of FFA from adipocytes into the conditioned medium were comparable between the cell lines (Figure 1C). Similar to a previous report (26), we also detected both aP2 and mal1 proteins in the conditioned medium from WT-Ad but not KO-Ad adipocytes (Supplemental Figure 1; supplemental material available online with this article; doi:10.1172/JCI34750DS1).

Having established these cellular systems, we first examined the impact of FABPs on monocyte chemoattractant protein-1 (MCP-1), a critical chemoattractant cytokine, in WT macrophages incubated with a conditioned medium obtained from either WT-Ad or KO-Ad differentiated adipocytes. Expression of $M c p 1$ was significantly lower in WT macrophages when incubated with the conditioned medium from FABP-deficient adipocytes compared with that from WT adipocytes (Figure 1D). This was also the case in FABP-deficient macrophages under the same experimental conditions (Figure 1D). To confirm this phenomenon in another setting, the same experiment was performed using primary macrophages exposed to conditioned medium from either KO+GFP-Ad (FABP-deficient) or $\mathrm{KO}+\mathrm{aP2}-\mathrm{Ad}$ (FABP-reconstituted) adipocytes. In both primary macrophages, WT-pMac and KO-pMac, expression of Mcp1 was significantly higher when they were incubated with the conditioned medium from $\mathrm{KO}+\mathrm{aP2}-\mathrm{Ad}$ than that from $\mathrm{KO}+\mathrm{GFP}-\mathrm{Ad}$ (Figure 1E). These results demonstrate that both the inflammatory output of adipocytes on macrophages and the inflammatory responsiveness of macrophages are regulated by FABPs.

To determine whether such interactions were reflected in the insulin sensitivity of adipocytes, we next constructed the reciprocal experiment, where we cocultured WT or FABP-deficient macrophages with differentiated 3T3-L1 adipocytes and examined the impact of macrophages on insulin action in adipocytes. When the adipocytes were exposed to WT macrophages, there was a marked suppression of insulin-stimulated glucose transport (about $75 \%$ reduction compared with control, untreated adipocytes) (Figure 1F). This suppressive effect was significantly diminished in the FABP-deficient macrophages. Coculturing the adipocytes with FABP-deficient macrophages did not result in full restoration of normal responsiveness to insulin. However, the suppressive impact of FABP-deficient macrophages, compared with WT macrophages, was markedly attenuated. We also examined insulin-stimulated Akt phosphorylation in this same setting to evaluate insulin signaling. Consistent with the glucose transport experiments, exposure of 3T3-L1 adipocytes to WT macrophages 
A

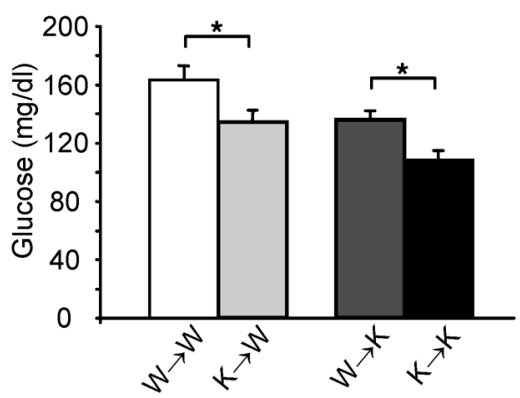

D

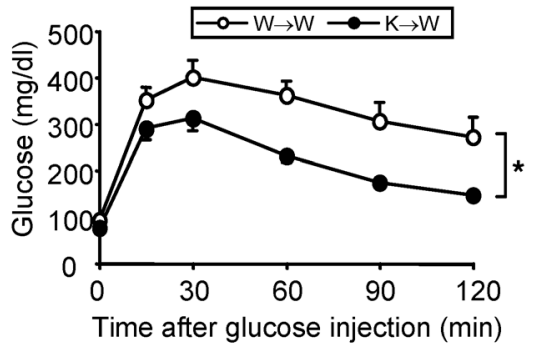

G

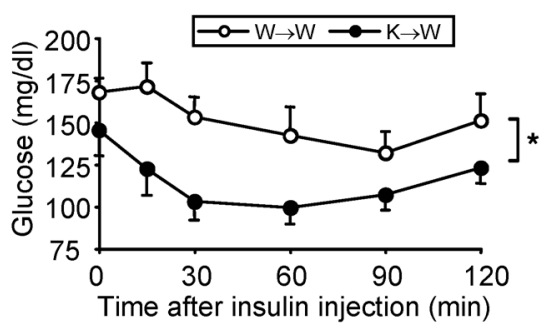

B

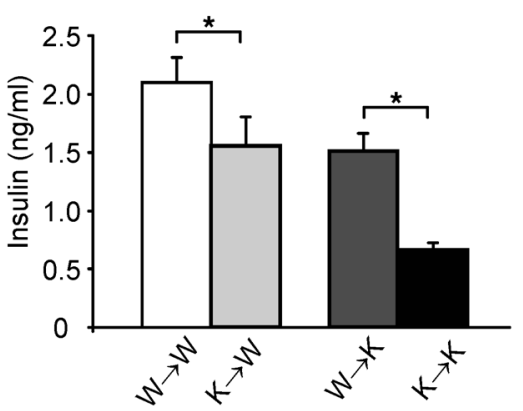

E

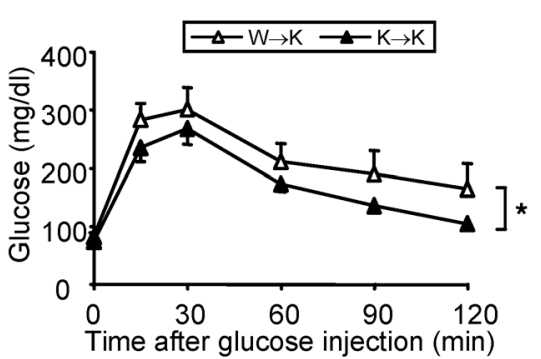

H

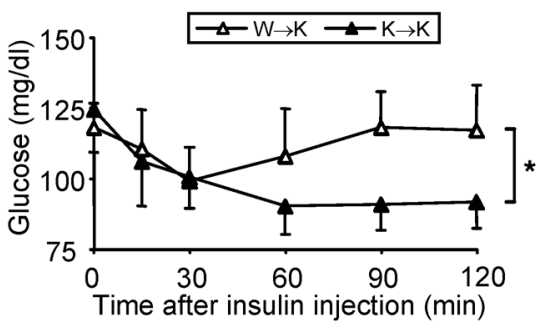

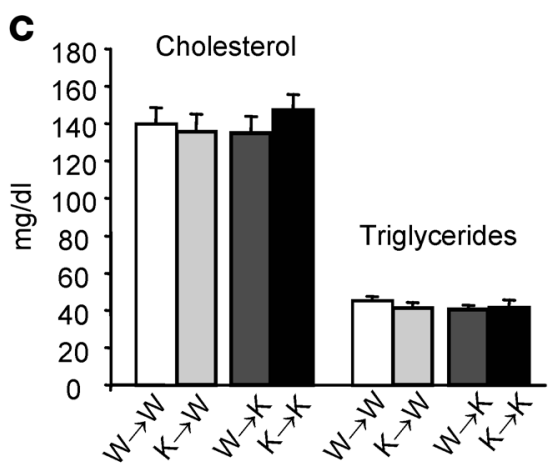

F
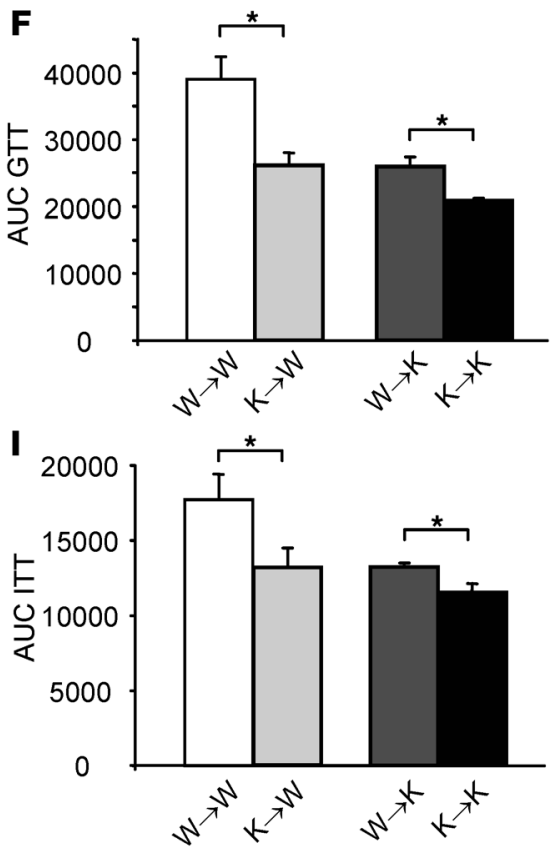

Figure 4

Glucose and lipid metabolism in BMT mice. (A-C) Levels of fasting blood glucose (A), insulin (B), and the lipid variables cholesterol and triglycerides (C) in BMT mice ( $\mathrm{W} \rightarrow \mathrm{W}, n=9 ; \mathrm{K} \rightarrow \mathrm{W}, n=10 ; \mathrm{W} \rightarrow \mathrm{K}, n=9$; and $\mathrm{K} \rightarrow \mathrm{K}, n=8)$ at 22 weeks of age. (D-F) Glucose tolerance tests performed in WT (D) and FABP-deficient (E) recipients at 19 weeks of age. (F) The AUC of glucose levels during tolerance tests is shown. (G-I) Insulin tolerance tests were performed in WT (G) and FABP-deficient $(\mathbf{H})$ recipients at 20 weeks of age. The AUC of glucose levels during tolerance tests is shown (I). Data are shown as mean \pm SEM. ${ }^{*} P<0.05$.

resulted in a marked suppression of insulin's ability to increase Akt phosphorylation (Figure 1G). The suppressive effect of macrophages on adipocyte insulin action was partially but significantly lost in FABP-deficient macrophages. We furthermore repeated this experiment using alternative experimental components. In this case, we utilized $\mathrm{KO}+\mathrm{GFP}-\mathrm{Ad}$ (FABP-deficient) or $\mathrm{KO}+\mathrm{aP} 2-\mathrm{Ad}$ (FABP-reconstituted) adipocytes and cultivated these cells with primary macrophages obtained from WT or FABP-deficient mice. The results demonstrated that the WT primary macrophages, WT-pMac, exhibited a greater capacity to interfere with insulin's ability to increase Akt phosphorylation in both adipocytes than the KO-pMac did (Figure 1H). Taken together, these findings demonstrate that FABPs are critical for regulating the inflammatory responses of macrophages and adipocytes and mediating the ability of macrophages to regulate insulin action in adipocytes. Accordingly, FABPs in both adipocytes and macrophages independently play a role in the development of insulin resistance.
Generation of radiation chimeras with adipocyte or macrophage FABP deficiency. Our observations in cellular systems prompted us to examine the FABP-mediated interactions between adipocytes and macrophages and their impact on systemic insulin sensitivity in vivo. Bone marrow transplantation (BMT) studies in gene-targeted mice have been a powerful tool to examine the contribution of specific pathways in macrophages to systemic diseases such as atherogenesis and could also constitute a valuable method for assessing the role of adipose tissue macrophages on insulin sensitivity. This experimental approach is particularly powerful when the target gene expression is enriched in or restricted to macrophages among bone marrow-derived cells and adipocytes in the stroma. Although it is hard to claim exclusivity, this largely applies to the lipid chaperone studies here, the joint action of which is highly restricted to adipocytes and macrophages. To evaluate the efficiency of our experimental system prior to performing BMT experiments with FABP mutants, we first transplanted bone mar- 
A

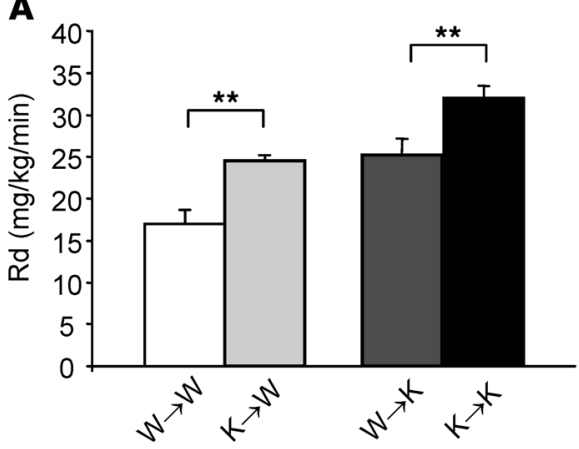

D

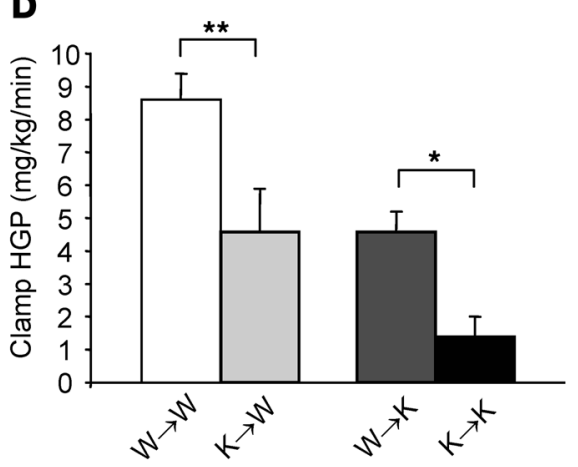

B

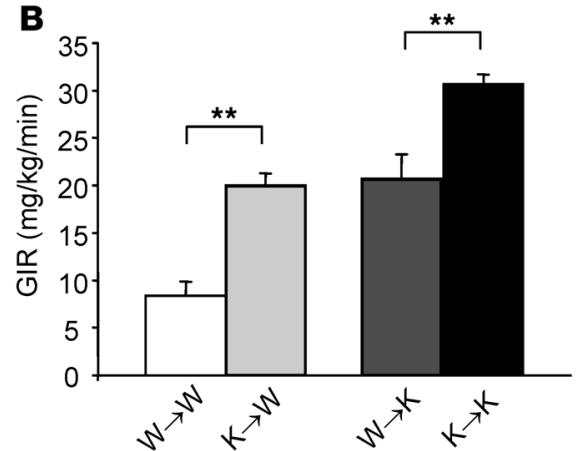

E

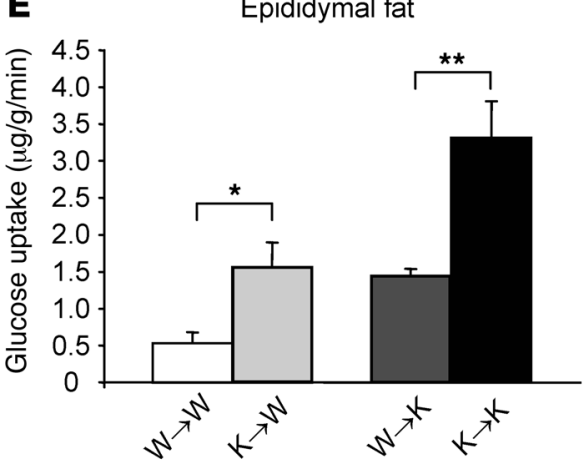

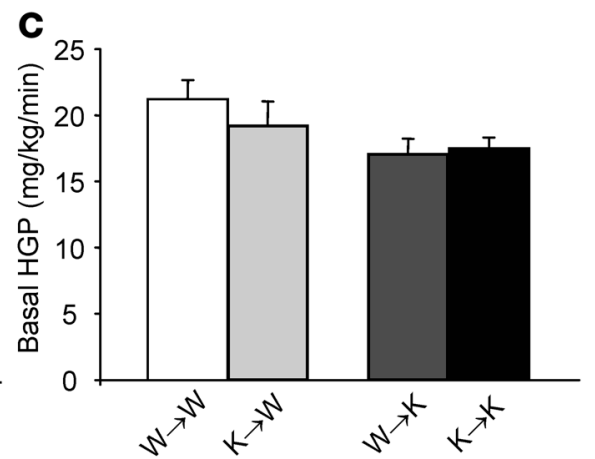

$\mathbf{F}$

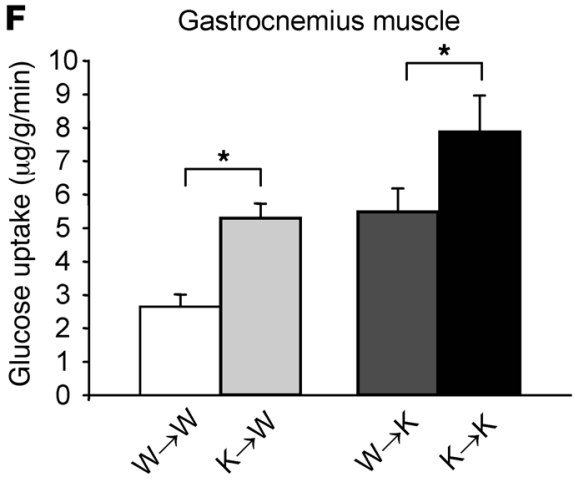

Figure 5

Hyperinsulinemic-euglycemic clamp in BMT mice. (A-F) Hyperinsulinemic-euglycemic clamp studies were performed in all groups of BMT mice $(\mathrm{W} \rightarrow \mathrm{W}, n=7 ; \mathrm{K} \rightarrow \mathrm{W}, n=5 ; \mathrm{W} \rightarrow \mathrm{K}, n=5 ;$ and $\mathrm{K} \rightarrow \mathrm{K}, n=7)$ at 20 weeks of age. Glucose disposal rate (Rd) (A), glucose infusion rate (GIR) (B), hepatic glucose production (HGP) at the basal (C) and clamp (D) states, and tissue glucose uptake in epididymal fat (E) and gastrocnemius muscle (F). Data are shown as mean \pm SEM. ${ }^{*} P<0.05,{ }^{* *} P<0.01$.

row cells from B6.SJL Ptprc $\mathrm{Pep}^{b} /$ BoyJ $\left(\mathrm{CD} 45.1^{+}\right)$donors into C57BL/6J (CD45.2 $\left.2^{+}\right)$recipient mice. Blood was collected from recipient mice 4 weeks after transplantation, and peripheral engraftment was confirmed using FACS analysis based on the presence of CD45.1 and absence of CD45.2 antigens in the resulting chimeras. This experiment demonstrated that approximately $98 \%$ of peripheral blood leukocytes in recipients were donor-derived $\mathrm{CD} 45.1^{+}$cells in our protocol. We also transplanted bone marrow cells from C57BL/6-Tg(UBC-GFP)30Scha/J donors, where all cells are labeled with GFP, into $\mathrm{C} 57 \mathrm{BL} / 6 \mathrm{~J}$ recipient mice to track the destination of the bone marrow-derived cells within adipose tissue. It has been suggested that bone marrow-derived cells could give rise to adipocytes under some but not all conditions $(27,28)$. Following BMT of GFP-labeled donor cells, we isolated the adipocyte and stromal-vascular (SV) fractions from the recipient adipose tissue. Expression of adiponectin and Pecam 1 was examined to confirm the proper fractionation of adipose tissue (Figure 2A). We also performed experiments to detect GFP mRNA and protein expression in the adipocyte and SV fractions. These experiments demonstrated that GFP was readily detectable in the SV fraction but not present in the adipocytes obtained from the GFP-transgenic BMT (GFP-Tg $\rightarrow$ WT) mice (Figure 2, B and C). By fluorescence microscopic analysis, we could not detect unilocular adipocytes in the adipocyte fraction of adipose tissue from the BMT $($ GFP-Tg $\rightarrow$ WT) mice (Figure 2D). The very few green cells detected in the adipocyte fraction were much smaller in size and did not appear as adipocytes, potentially representing contaminating cells from the SV fraction that contained GFP-labeled cells (Sup- plemental Figure 2). Taken together, our results indicate that the potential contribution of bone marrow-derived cells to adipocytes is not a confounding factor in the BMT experiments used in this study. Finally, we performed additional control experiments to confirm that expression of aP2 and mal1 was mainly restricted to the monocyte/macrophage $\left(\mathrm{CD} 14^{+}\right)$fraction in blood cells (Supplemental Figure 3, A-C) and that the expression was not detectable in $\mathrm{T}\left(\mathrm{CD}^{+}\right.$or $\left.\mathrm{CD}^{+}\right)$or $\mathrm{B}\left(\mathrm{CD} 19^{+}\right)$cells (data not shown).

Having obtained these results, we created mice chimeric for macrophage or adipocyte FABP deficiency, along with WT and FABP-deficient controls using BMT (Figure 3, A and B). All groups of mice were fed a high-fat diet from 4 weeks of age. Either WT or FABP-knockout bone marrow was collected and transplanted into 6-week-old recipient WT mice that had been lethally irradiated (W $\rightarrow W$ and $K \rightarrow W$, respectively). Similarly, FABP-knockout mice were lethally irradiated and transplanted with either WT (W $\rightarrow \mathrm{K}$ ) or FABP-knockout $(\mathrm{K} \rightarrow \mathrm{K})$ bone marrow. In these animals, we examined the extent of chimerism by genotyping for the WT and FABP-deficient alleles using genomic DNA extracted from blood and stromal tissue (tail) and confirmed more than $95 \%$ reconstitution of bone marrow-derived blood cells in all of the groups (Figure 3C). In addition, we also genotyped adipose tissue, liver, and skeletal muscle in all of the chimeric mice and verified the presence of donor cells represented by the donor genotype in these tissues (Figure 3D).

Metabolic studies in chimeric mice with adipocyte or macrophage FABP deficiency. Having confirmed the successful production of radiation chimeras, we proceeded with detailed metabolic examination in all 
A
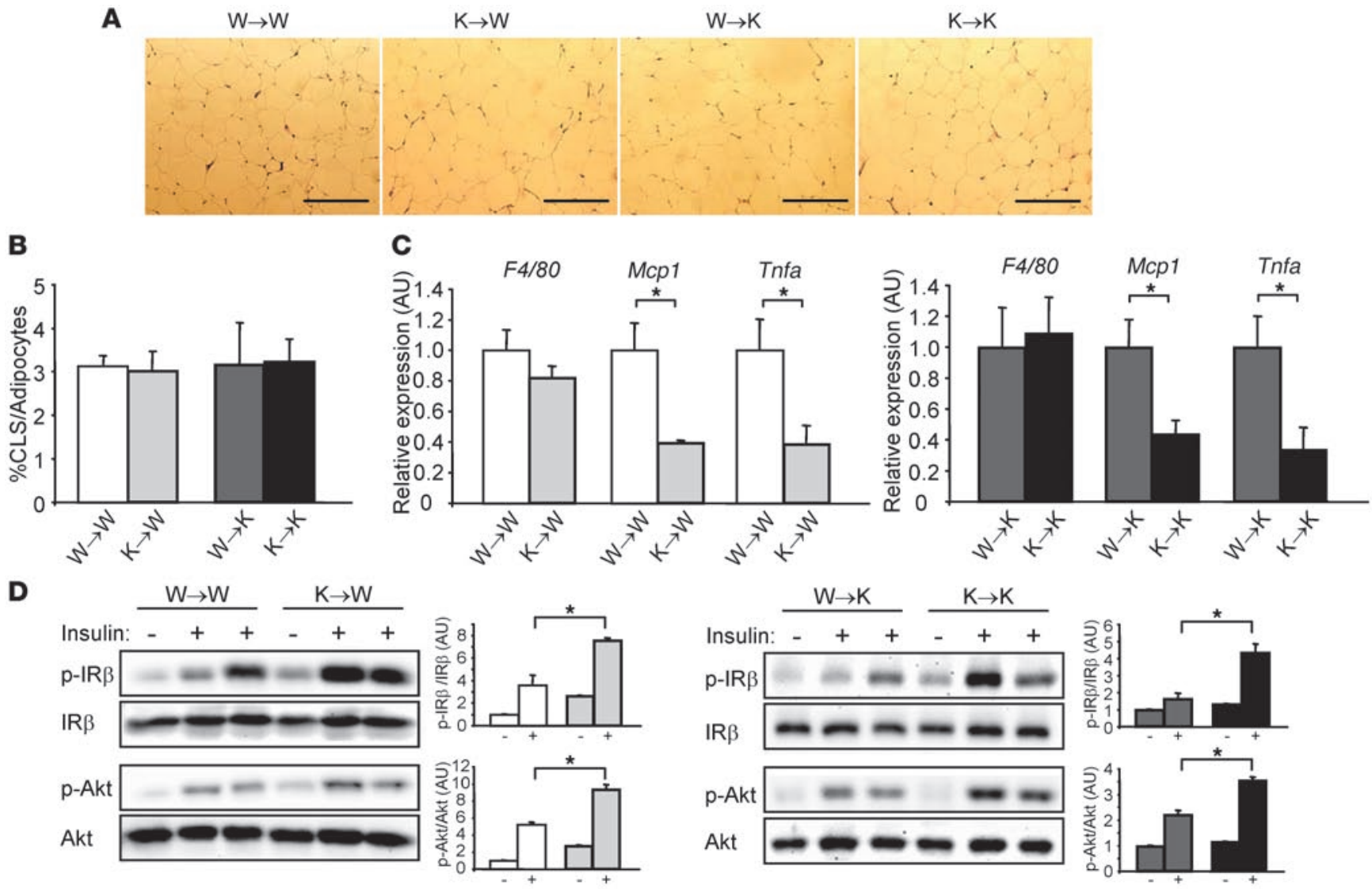

Figure 6

Adipose tissue characteristics and insulin action in BMT mice. (A) H\&E staining of the adipose tissue in BMT mice. Scale bars: $200 \mu \mathrm{m}$. (B) Adipose tissue macrophage content expressed as percentage of crown-like structure (CLS) detected by F4/80 staining per adipocyte. (C) Expression of F4/80, Mcp1, and Tnfa in the adipose tissue of BMT mice. (D) Insulin-stimulated IR $\beta$ tyrosine 1162/1163 and Akt serine 473 phosphorylation in the adipose tissues of BMT mice. The graphs to the right of the blots show the quantification from experiments performed at least in duplicate. Data are shown as mean \pm SEM. ${ }^{*} P<0.05$.

4 groups of mice. During the course of the experimental protocol until 24 weeks of age, there was no significant difference in body weight or total body adiposity among BMT animals (Supplemental Figure 4, A and B). Similarly, rates of oxygen consumption $\left(\mathrm{VO}_{2}\right)$ and carbon dioxide production $\left(\mathrm{VCO}_{2}\right)$, food intake, and physical activity levels were not different among the 4 groups of mice (Supplemental Figure 4, C-F). These results demonstrate that FABP chimerism does not impact weight or energy homeostasis and will allow examination of potential metabolic changes independent of body weight or adiposity. Next, we initiated studies to evaluate glucose metabolism and insulin sensitivity. In contrast to body weight and adiposity, levels of fasting blood glucose and insulin were significantly decreased in mice receiving FABP-deficient bone marrow compared with those reconstituted with bone marrow from WT donors (Figure 4, A and B). In both of these experiments, the impact of stromal FABP deficiency was greater than that in the bone marrow-derived compartment, but the results nevertheless indicated a potential contribution from the bone marrow-derived compartment to systemic insulin action. In these groups of mice, we did not observe a significant difference in serum lipid profiles, with similar levels of cholesterol and triglycerides among genotypes (Figure 4C). The serum levels of adiponectin in FABP-defi- cient recipient groups were lower than those in the WT recipient groups (Supplemental Figure 5), consistent with the earlier observations in total FABP-deficient mice (17). However, there was no significant difference in serum adiponectin levels between the 2 chimeric groups (Supplemental Figure 5).

We next performed glucose and insulin tolerance tests to examine systemic glucose metabolism and insulin action in all groups of mice. In support of the improvement in glucose metabolism by FABP deficiency in either macrophage or adipocyte compartments, glucose tolerance tests revealed a significant improvement in glucose disposal curves in mice receiving FABP-deficient bone marrow compared with those reconstituted with bone marrow from WT donors in both WT and FABP-deficient recipients (Figure 4, D-F). Similarly, insulin tolerance tests showed significantly increased insulin sensitivity in mice of either genotype receiving FABP-deficient bone marrow-derived elements (Figure 4, G-I). Despite a lower magnitude of weight gain following BMT (compared with nontransplanted mice on a high-fat diet), the absolute values of these metabolic parameters were essentially identical to those in nonirradiated WT or FABP-deficient mice as previously reported (17), indicating that the protocol used in our study induced comparable insulin resistance. These data demonstrate 
A
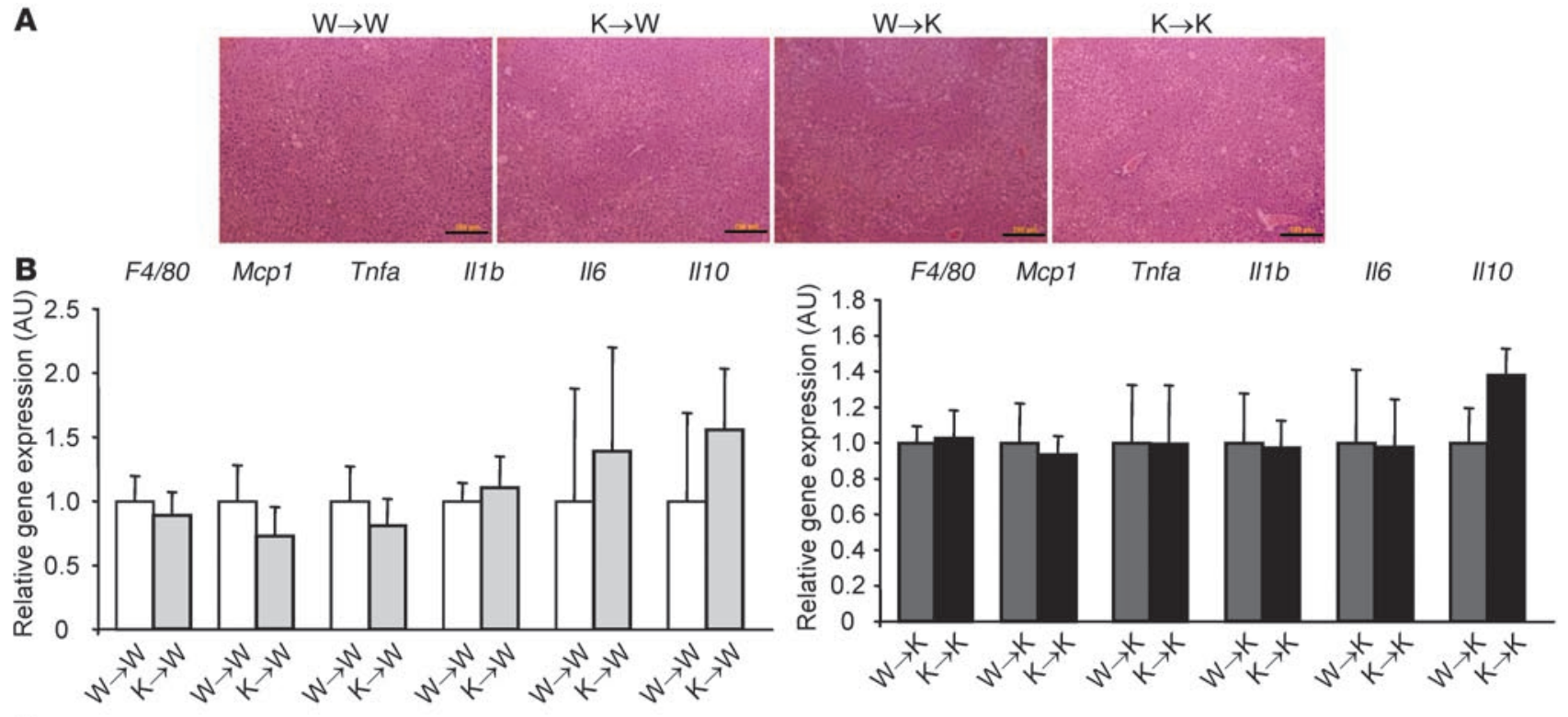

C
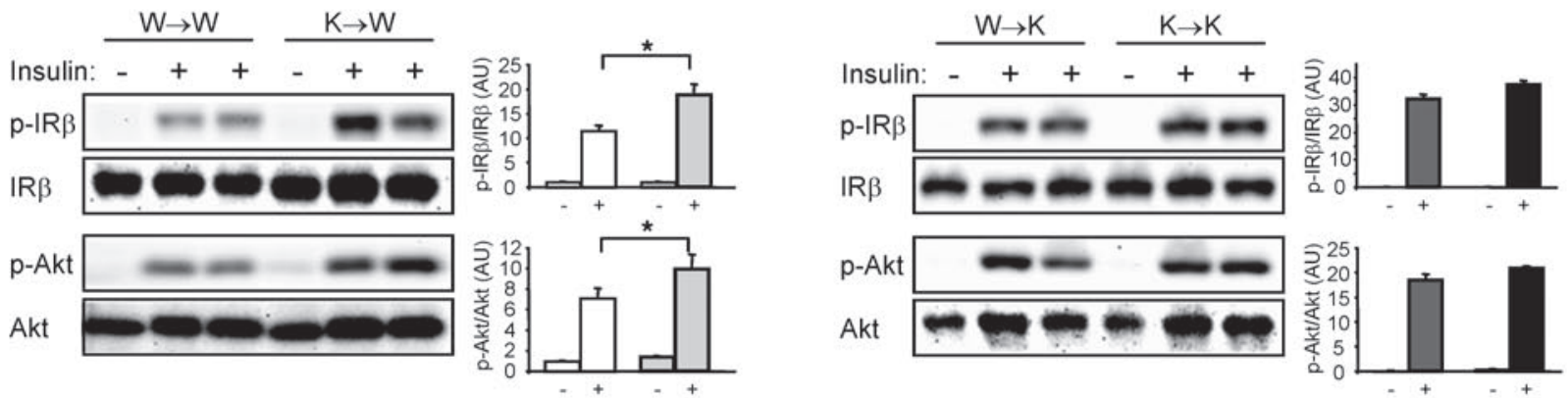

Figure 7

Liver tissue properties and insulin action in BMT mice. (A) H\&E staining of the liver sections in all groups of BMT mice. Scale bars: $200 \mu \mathrm{m}$. (B) Expression of $F 4 / 80, M c p 1$, Tnfa, I/1b, //6, and I/10 in the liver of BMT mice. (C) Insulin-stimulated IR $\beta$ tyrosine $1162 / 1163$ and Akt serine 473 phosphorylation in the liver tissues of BMT mice. The graphs to the right of the blots show the quantification from experiments performed at least in duplicate. Data are shown as mean \pm SEM. ${ }^{*} P<0.05$.

that FABP action in both macrophages and adipocytes contributes to the regulation of glucose metabolism, although the contribution from adipocytes appeared to be dominant, and these effects are independent of changes in body weight and composition or alterations in lipid metabolism in this experimental setting.

Whole-body glucose flux studies in chimeric mice. To determine the sites of change in systemic insulin sensitivity and glucose flux with more sensitivity and precision, we performed a hyperinsulinemiceuglycemic clamp study in mice 14 weeks after the BMT at the age of 20 weeks. Whole-body glucose disposal and glucose infusion rates were significantly increased after BMT with FABP-deficient cells compared with WT controls of either genotype (Figure 5, A and $\mathrm{B}$ ), demonstrating increased whole-body and peripheral insulin sensitivity. We also evaluated the impact of insulin in regulating hepatic glucose metabolism. There was no significant difference in basal hepatic glucose production (HGP) among the groups (Figure 5C). However, during the hyperinsulinemic clamp, HGP was suppressed more significantly in FABP-deficient bone marrow-transplanted mice compared with WT bone marrow-transplanted mice (Figure 5D). These data demonstrate that macrophage FABP action contributed to the regulation of whole-body insulin sensitivity via both suppression of HGP and enhancement of insulin-stimulated glucose disposal in peripheral tissues, although this impact was not the major determinant of whole-body insulin sensitivity. To explore this further, we determined the rate of glucose uptake specifically in epididymal adipose tissue and gastrocnemius muscle during the clamp procedure. In mice of either genotype receiving FABP-deficient bone marrow, glucose uptake in the adipose tissue and muscle was significantly increased compared with that in mice reconstituted with the WT bone marrow (Figure 5, E and F). The insulin-stimulated glucose uptake was the highest in FABPdeficient mice receiving FABP-deficient bone marrow and lowest in WT mice receiving WT bone marrow. The remaining 2 chimeras exhibited an intermediary phenotype, demonstrating that FABP deficiency in macrophages (bone marrow) or adipocytes (stroma) each partially contributed to the effects of FABPs on systemic insulin action. This pattern was consistent with our metabolic studies and also reproduced in the changes in whole-body glucose flux and hepatic glucose output (Figures 4 and 5).

Adipose tissue in adipocyte or macrophage FABP deficiency. Since adipose tissue is the major site of the coexpression of adipocytes and macrophages, we next examined whether the inflammatory status of adipose tissue or insulin action is altered depending on the source of FABP deficiency at this site. In histological studies, adi- 
pose tissue appeared similar in all 4 groups of mice. Adipocyte size and morphology were comparable between the genotypes (Figure $6 \mathrm{~A}$ ). Adipose tissue macrophage infiltration was evaluated by both immunohistochemistry and analysis of the expression level of the commonly used macrophage marker F4/80. The presence of cells recognized with this marker was evident in adipose tissue; however, the extent of F4/80-positive cellular infiltration as assessed by the presence of crown-like structures or the level of mRNA expression did not differ significantly among the groups (Figure 6, B and C). Hence, FABP deficiency created in either macrophages or adipocytes did not have a major impact on the number of macrophages present in adipose tissue under the experimental conditions used in this study. Since inflammation in adipose tissue is critical for metabolic pathologies (1), we next determined the expression of chemoattractant and inflammatory cytokines, MCP- 1 and TNF- $\alpha$, in adipose tissue to evaluate the inflammatory output of the tissue. Interestingly, expression of both Mcp1 and Tnfa in adipose tissue was significantly reduced in the FABP-deficient bone marrowtransplanted mice compared with WT bone marrow-transplanted mice (Figure 6C), demonstrating that FABP action in both cellular targets in adipose tissue, macrophages and adipocytes, contributes to the inflammatory responses. The change in inflammatory mediators in adipose tissue was not a general feature for all mediators, and no significant difference was evident in the expression of $I l 6$ and Illb among chimeras (Supplemental Figure 6). There was also no significant difference in expression levels of Il10, an antiinflammatory cytokine, and adiponectin in adipose tissue between the groups (Supplemental Figure 6).

We next examined whether the alteration in inflammatory status of adipose tissue macrophages or adipocytes in the absence of FABPs resulted in a change in insulin action at this site. To evaluate this, we biochemically examined adipose tissue insulin receptor signaling capacity in intact mice following insulin administration. Insulin-stimulated tyrosine $1162 / 1163$ phosphorylation of IR $\beta$ subunit (IR $\beta$ ) and serine 473 phosphorylation of Akt were significantly increased in the adipose tissue of WT or FABP-deficient mice receiving FABP-deficient bone marrow compared with those reconstituted with WT bone marrow (Figure 6D). These results demonstrate that $\mathrm{FABP}$ deficiency in either macrophages or adipocytes increases insulin sensitivity and receptor signaling capacity in adipose tissue.

We also examined livers in all 4 groups of mice for morphological alterations, the extent of fatty infiltration, and insulin action. H\&E staining of liver sections did not reveal a significant difference in lipid infiltration among groups (Figure 7A). The expression of macrophage and inflammatory markers in liver were not different among the groups (Figure 7B). Insulin-stimulated phosphorylation of IR $\beta$ and Akt in liver was significantly higher in $\mathrm{K} \rightarrow \mathrm{W}$ than in $\mathrm{W} \rightarrow \mathrm{W}$ mice, but no significant difference was observed between $\mathrm{W} \rightarrow \mathrm{K}$ and $\mathrm{K} \rightarrow \mathrm{K}$ mice (Figure $7 \mathrm{C}$ ). In the FABP-deficient recipient group, insulin resistance did not appear to be severe, regardless of the source of the transplanted bone marrow. Finally, we analyzed expression levels of cytokines in skeletal muscle, and only in the muscle tissue of FABP-deficient recipient groups did we find differences in the levels of Mcp1 and Il6 (Supplemental Figure 7).

\section{Discussion}

Several lines of evidence have clearly shown that obesity causes a state of chronic, low-grade inflammation that contributes to insulin resistance and type 2 diabetes (1). It has also been suggested that accumulation of immune cells, particularly macrophages, in adipose tissue may be an important component of the development of adipose tissue inflammatory responses triggered by obesity and hence contribute to the metabolic consequences, such as insulin resistance $(2,3)$. Since macrophages are potent producers of many inflammatory mediators, they are likely to contribute to the inflammatory responses seen in obesity. However, the extent of this contribution and the impact on systemic or local insulin action is not yet clear. A study in myeloid-specific deletion of IкB kinase $\beta$, an important regulator of inflammatory response through activation of NF-кB, showed improvement in whole-body insulin sensitivity in mice on a high-fat diet (8). While the inflammatory impact of this genetic intervention or the precise role of targeted cell populations on adipose tissue is not yet clear (29), this study provided strong evidence supporting the involvement of bone marrow-derived cells in insulin sensitivity. There have also been attempts to promote or reduce macrophage infiltration through manipulation of macrophage chemoattractant molecules or their signaling receptors (30-33). While some of these studies have generated important insights, a cohesive picture has not yet emerged due to the widespread action of targeted molecules and variations in the phenotypes observed in these genetic models. There are still important gaps in the understanding of functional consequences of macrophage infiltration into adipose tissue during obesity, the potential interactions between macrophages and adipocytes, and the pathways involved in these interactions.

A critical mechanism linking inflammatory and metabolic responses involves the action of adipocyte/macrophage FABPs. The total deletion of these FABPs produces a marked resistance to pathologies associated with dietary or genetic obesity, including insulin resistance, type 2 diabetes, and atherosclerosis (17-19). Recent studies also demonstrated the potential relevance of this mechanism to type 2 diabetes and cardiovascular disease in humans $(20,21)$. In a previous study, we have demonstrated that the impact of aP2 on atherosclerosis was essentially exclusive to its actions in macrophages (12). However, whether FABP action in macrophages also contributes to the systemic insulin action was unknown. In the present study, based on the biology and cellular distribution of FABPs, we have postulated that this model may offer some advantages for exploring the interactions between adipose tissue macrophages and adipocytes in metabolic diseases and might allow for dissection of the role of each target cell in inflammatory and metabolic alterations associated with obesity. This model is also suitable for performing BMT studies to examine macrophage- or adipocyte-restricted FABP deficiency, as those are the major sites of FABP coexpression. In the BMT protocol used in our study, we did not see a contribution of bone marrow-derived cells to the formation of adipocytes. Hence, the metabolic effects could not be attributed to transdifferentiating adipocytes originating from the donor cells. Furthermore, following BMT, there was no difference in body weight of the chimeric mice, allowing the comparisons independent of changes in body weight or systemic adiposity, a common phenotype seen in many BMT studies. It is worth mentioning that the maximum weight gain was lower in mice that underwent BMT than in intact mice fed a high-fat diet. However, despite lower overall weight gain, metabolic deterioration is comparable.

In this setting, FABP deficiency in bone marrow-derived elements led to significant protection from the development of insulin resistance in WT recipient mice with intact adipocytes. In the reciprocal experiments, absence of FABP expression in recipient 
adipocytes reconstituted with WT bone marrow partially decreased insulin sensitivity. Both macrophage (bone marrow)- and adipocyte (stroma)-restricted FABP deficiency inhibited expression of inflammatory cytokines and ameliorated insulin action and signaling in the adipose tissue of mice kept on a high-fat diet. Taken together, these results indicate the contributions of macrophages and adipocytes to obesity-related inflammatory changes in adipose tissue and insulin resistance. Experiments using coculture systems with several macrophage and adipocyte cell lines showed that deletion of FABPs in adipocytes reduced expression of inflammatory cytokines in macrophages, and the same deletion in macrophages enhanced insulin signaling and glucose uptake in adipocytes. Based on these data, a potential mechanism underlying the metabolic syndrome emerges from coordinated modulation of the metabolic status in adipocytes and the inflammatory status in macrophages through pathways common to both cell types controlled by FABPs.

Our findings demonstrate that FABPs control the development of major components of the metabolic syndrome through their distinct actions in adipocytes and macrophages independently. These results are of significance for several reasons. First, our data demonstrate that the contribution of macrophages in obesityinduced adipose tissue inflammation and insulin resistance is significant but partial. It is also clear from the present study that adipocytes are significant contributors to inflammatory alterations in adipose tissue leading to both local and systemic metabolic deterioration. Interestingly, we did not observe dramatic alterations in the amount of adipose tissue macrophages in any one of the groups. This observation suggests that the presence of macrophages per se is not a direct indicator of metabolic dysfunction and that the signals emanating from the local adipocytes in contact with macrophages and their activation state are more critical aspects of their impact on adipose tissue function. The metabolic status of adipocytes is therefore a major determinant of macrophage inflammatory output. In fact, the impact of FABP action in adipocytes (or stromal elements) on overall metabolic health appeared to be dominant in this setting. It remains to be seen whether adipocyte-derived signals regulated by these FABPs also play a role in the metabolic alterations and enhanced insulin action seen in liver or muscle tissues.

Second, our findings may stimulate important considerations regarding the therapeutic development and utilization of synthetic aP2 inhibitors. Since aP2 is expressed at very high levels in adipocytes, it has been questioned whether the efficacy of aP2 inhibitors in adipocytes would be enough to compete with aP2 binding to lipids. A recent study demonstrated that an orally active and specific aP2 inhibitor is highly effective for treatment of both atherosclerosis and type 2 diabetes in independent mouse models (22). In these inhibitor studies, a higher dose of aP2 inhibitor was required for the treatment of diabetes compared with atherosclerosis (22). Taken together with those of the present study, the results indicate that the efficacy against type 2 diabetes, similar to atherosclerosis, might be related to the regulation of FABP function in macrophages. Since the aP2 action in macrophages is responsible for the entire phenotype in atherosclerosis (12), the synthetic inhibitor was extremely effective in this paradigm, indicating that chemical FABP inhibitors can target macrophages very effectively. Similarly, since macrophages also contribute to insulin resistance, it may be possible to treat type 2 diabetes through FABP inhibition, potentially even without targeting (or only partial targeting) of adipo- cytes, through the complementary action in macrophages. In light of emerging human data showing the impact of aP2 in type 2 diabetes and atherosclerosis, it is possible that targeting this pathway in humans could provide novel therapeutic opportunities.

\section{Methods}

Reagents and animals. All biochemical reagents were purchased from Sigma-Aldrich unless otherwise indicated. Animal care and experimental procedures were performed with approval from the Harvard Medical School IACUC. B6.SJL Ptprc ${ }^{a} P e p 3^{b} /$ BoyJ (CD45.1 $)$, C57BL/6J (CD45.2 $)$, and $\mathrm{C} 57 \mathrm{BL} / 6-\mathrm{Tg}(\mathrm{UBC}-\mathrm{GFP}) 30 \mathrm{Sch} / \mathrm{J}$ mice were purchased from The Jackson Laboratory. Mice deficient in both aP2 and mal1 were generated as previously described (17). The mice were backcrossed more than 12 generations into the $\mathrm{C} 57 \mathrm{BL} / 6 \mathrm{~J}$ genetic background. Both WT

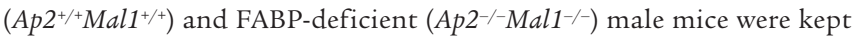
on a 12-hour-light /12-hour-dark cycle in a pathogen-free barrier facility and were placed on a high-fat diet (D12492: kcal\% of fat, $60 \%$; Research Diets), ad libitum, beginning at 4 weeks of age.

$B M T$. Six-week-old recipient mice were lethally irradiated with two 5-Gy doses (total $10 \mathrm{~Gy}$ ) from a cesium source at a 4-hour interval in order to minimize radiation toxicity. Bone marrow was collected by flushing the femurs and tibias from sex-matched donor mice ( $6-8$ weeks of age) into Gibco RPMI 1640 medium (Invitrogen). Four hours after the second irradiation, $5 \times 10^{6}$ bone marrow cells in $0.2 \mathrm{ml}$ were injected in the retro-orbital venous plexus. During 1 week before and 4 weeks following BMT, $100 \mathrm{mg} / \mathrm{l}$ neomycin and $10 \mathrm{mg} / \mathrm{l}$ polymyxin $\mathrm{B}$ sulfate were added to the acidified water.

Fluorescence microscopy in GFP-transgenic BMT mice. Bone marrow cells from C57BL/6-Tg(UBC-GFP)30Scha/J mice were transplanted into WT recipients. Adipocyte and SV fractions were isolated from the BMT (GFP-Tg $\rightarrow$ WT) mice that were on a high-fat diet for 25 weeks after BMT. GFP signal was detected by a fluorescence microscope (AxioVision; Zeiss) using filter set $38 \mathrm{HE}$ (excitation, BP 470/40; beam splitter, FT 495 HE; emission, BP $525 / 50 \mathrm{HE}$ ). As controls, the fractions isolated from WT and GFP-transgenic mice were also analyzed. For the adipocyte fraction, the exposure time of centrifuged and floating preparations was 30 and $100 \mathrm{~ms}$, respectively. For the SV fraction, exposure time of low and high magnifications was 100 and $200 \mathrm{~ms}$, respectively.

Genotyping. Samples of blood or tissue were lysed with nonionic detergents and proteinase $\mathrm{K}$, and DNA was isolated using phenol and chloroform. PCR was performed to determine the genotype of aP2 using 3 specific primers (5'-CAGCACTCACCCACTTCTTTCAT-3', 5'-ACATACAGGGTC TGGTG-3', and 5'-ATAGCAGCCAGTCCCTTCCCGCTT-3'). Aliquots of the reaction product were analyzed by electrophoresis in $2 \%$ agarose gels containing ethidium bromide and visualized under UV light.

Coculture experiments. A conditioned medium from adipocytes was prepared as supernatants from adipocytes incubated in DMEM supplemented with $0.5 \%$ BSA for 16 hours. After filtering the supernatants, lipoproteindeficient serum (final concentration, $5 \%$ ) was added in the filtered supernatants. Macrophages were incubated in the conditioned medium for 24 hours. For insulin action experiments, $1 \times 10^{5}$ macrophages were directly added onto adipocytes in a 12 -well plate. The cells were incubated in DMEM supplemented with heat-inactivated $10 \%$ cosmic calf serum for 24 hours and then in DMEM with $0.5 \%$ BSA overnight, followed by treatment with $100 \mathrm{nM}$ insulin for 3 minutes. Each experiment was done at least in triplicate.

Quantitative real-time PCR analysis. Total RNA was isolated using TRIzOL reagent (Invitrogen). For reverse transcription, $1 \mu \mathrm{g}$ of the total RNA was converted to first-strand cDNAs in $20 \mu \mathrm{l}$ reactions using a High-Capacity cDNA Archive Kit (Applied Biosystems). Quantitative real-time PCR analysis was performed using SYBR Green in a real-time PCR system (Applied Biosystems). The thermal cycling program was 10 minutes at $95^{\circ} \mathrm{C}$ for 
enzyme activation and 40 cycles of denaturation for 15 seconds at $95^{\circ} \mathrm{C}$, 30 -second annealing at $58^{\circ} \mathrm{C}$, and 30 -second extension at $72^{\circ} \mathrm{C}$. Primers used in the present study are listed in Supplemental Table 1. To normalize expression data, 18s rRNA was used as an internal control gene.

Statistics. Experimental results are shown as mean \pm SEM. After analysis of distribution normality using the Shapiro-Wilk $W$ test, the mean values for biochemical data from each group were compared by Student's $t$ test. Comparisons between time points were analyzed using repeated-measures ANOVA. Statistical tests with $P<0.05$ were considered significant.

\section{Acknowledgments}

This work was supported in part by grants from the NIH (DK52540 and HL65405) and the American Diabetes Association. M. Furuhashi is supported by fellowships from the Japan Society for the Pro- motion of Science and the American Diabetes Association. G. Tuncman is supported by a fellowship from the Iacocca Foundation. $\mathrm{H}$. Cao is supported by an NIH Roadmap Fellowship (DK71507-04). We thank Güllü Görgün of the Dana-Farber Cancer Institute for help in isolation of $\mathrm{CD} 14^{+}$fractions from blood samples.

Received for publication December 13, 2007, and accepted in revised form May 7, 2008.

Address correspondence to: Gökhan S. Hotamisligil, Department of Genetics and Complex Diseases, Harvard School of Public Health, 665 Huntington Avenue, Boston, Massachusetts 02115, USA. Phone: (617) 432-1950; Fax: (617) 432-1941; E-mail: ghotamis@ hsph.harvard.edu.
1. Hotamisligil, G.S. 2006. Inflammation and metabolic disorders. Nature. 444:860-867.

2. Xu, H., et al. 2003. Chronic inflammation in fat plays a crucial role in the development of obesity-related insulin resistance. J. Clin. Invest. 112:1821-1830

3. Weisberg, S.P., et al. 2003. Obesity is associated with macrophage accumulation in adipose tissue. J. Clin. Invest. 112:1796-1808.

4. Wellen, K.E., and Hotamisligil, G.S. 2005. Inflammation, stress, and diabetes. J. Clin. Invest. 115:1111-1119.

5. Cinti, S., et al. 2005. Adipocyte death defines macrophage localization and function in adipose tissue of obese mice and humans. J. Lipid Res. 46:2347-2355.

6. Lesniewski, L.A., et al. 2007. Bone marrow-specific Cap gene deletion protects against high-fat dietinduced insulin resistance. Nat. Med. 13:455-462.

7. Solinas, G., et al. 2007. JNK1 in hematopoietically derived cells contributes to diet-induced inflammation and insulin resistance without affecting obesity. Cell Metab. 6:386-397.

8. Arkan, M.C., et al. 2005. IKK- $\beta$ links inflammation to obesity-induced insulin resistance. Nat. Med. 11:191-198.

9. Odegaard, J.I., et al. 2007. Macrophage-specific PPAR $\gamma$ controls alternative activation and improves insulin resistance. Nature. 447:1116-1120.

10. Furuhashi, M., and Hotamisligil, G.S. 2008. Fatty acid-binding proteins: role in metabloic diseases and potential as drug targets. Nat Rev. Drug Discov. 7:489-503.

11. Hunt, C.R., Ro, J.H., Dobson, D.E., Min, H.Y., and Spiegelman, B.M. 1986. Adipocyte P2 gene: developmental expression and homology of 5 '-flanking sequences among fat cell-specific genes. Proc. Natl. Acad. Sci. U. S. A. 83:3786-3790.

12. Makowski, L., et al. 2001. Lack of macrophage fattyacid-binding protein $\mathrm{aP} 2$ protects mice deficient in apolipoprotein E against atherosclerosis. Nat. Med. 7:699-705

13. Makowski, L., Brittingham, K.C., Reynolds, J.M.,
Suttles, J., and Hotamisligil, G.S. 2005. The fatty acid-binding protein, aP2, coordinates macrophage cholesterol trafficking and inflammatory activity. Macrophage expression of aP2 impacts peroxisome proliferator-activated receptor $\gamma$ and IкB kinase activities. J. Biol. Chem. 280:12888-12895.

14. Hotamisligil, G.S., et al. 1996. Uncoupling of obesity from insulin resistance through a targeted mutation in aP2, the adipocyte fatty acid binding protein. Science. 274:1377-1379.

15. Uysal, K.T., Scheja, L., Wiesbrock, S.M., BonnerWeir, S., and Hotamisligil, G.S. 2000. Improved glucose and lipid metabolism in genetically obese mice lacking aP2. Endocrinology. 141:3388-3396.

16. Maeda, K., et al. 2003. Role of the fatty acid binding protein mal1 in obesity and insulin resistance. Diabetes. 52:300-307.

17. Maeda, K., et al. 2005. Adipocyte/macrophage fatty acid binding proteins control integrated metabolic responses in obesity and diabetes. Cell Metab. 1:107-119.

18. Cao, H., et al. 2006. Regulation of metabolic responses by adipocyte/macrophage Fatty Acidbinding proteins in leptin-deficient mice. Diabetes. 55:1915-1922.

19. Boord, J.B., et al. 2004. Combined adipocyte-macrophage fatty acid-binding protein deficiency improves metabolism, atherosclerosis, and survival in apolipoprotein E-deficient mice. Circulation. 110:1492-1498.

20. Tuncman, G., et al. 2006. A genetic variant at the fatty acid-binding protein aP2 locus reduces the risk for hypertriglyceridemia, type 2 diabetes, and cardiovascular disease. Proc. Natl. Acad. Sci. U. S. A. 103:6970-6975.

21. Fu, Y., Luo, N., Lopes-Virella, M.F., and Garvey, W.T. 2002. The adipocyte lipid binding protein (ALBP/aP2) gene facilitates foam cell formation in human THP-1 macrophages. Atherosclerosis. 165:259-269.

22. Furuhashi, M., et al. 2007. Treatment of diabetes and atherosclerosis by inhibiting fatty-acid-binding protein aP2. Nature. 447:959-965.
23. Shum, B.O., et al. 2006. The adipocyte fatty acidbinding protein aP2 is required in allergic airway inflammation. J. Clin. Invest. 116:2183-2192.

24. Scheja, L., et al. 1999. Altered insulin secretion associated with reduced lipolytic efficiency in aP2-/mice. Diabetes. 48:1987-1994.

25. Coe, N.R., Simpson, M.A., and Bernlohr, D.A. 1999. Targeted disruption of the adipocyte lipid-binding protein (aP2 protein) gene impairs fat cell lipolysis and increases cellular fatty acid levels. J. Lipid Res. 40:967-972.

26. Xu, A., et al. 2006. Adipocyte fatty acid-binding protein is a plasma biomarker closely associated with obesity and metabolic syndrome. Clin. Chem. 52:405-413.

27. Crossno, J.T., Majka, S.M., Grazia, T., Gill, R.G., and Klemm, D.J. 2006. Rosiglitazone promotes development of a novel adipocyte population from bone marrow-derived circulating progenitor cells. J. Clin. Invest. 116:3220-3228.

28. Koh, Y.J., et al. 2007. Bone marrow-derived circulating progenitor cells fail to transdifferentiate into adipocytes in adult adipose tissues in mice. J. Clin. Invest. 117:3684-3695.

29. Clausen, B.E., Burkhardt, C., Reith, W., Renkawitz, R., and Förster, I. 1999. Conditional gene targeting in macrophages and granulocytes using LysMcre mice. Transgenic Res. 8:265-277.

30. Kanda, H., et al. 2006. MCP-1 contributes to macrophage infiltration into adipose tissue, insulin resistance, and hepatic steatosis in obesity. J. Clin. Invest. 116:1494-1505.

31. Kamei, N., et al. 2006. Overexpression of monocyte chemoattractant protein-1 in adipose tissues causes macrophage recruitment and insulin resistance. J. Biol. Chem. 281:26602-26614.

32. Weisberg, S.P., et al. 2006. CCR2 modulates inflammatory and metabolic effects of high-fat feeding. J. Clin. Invest. 116:115-124.

33. Inouye, K.E., et al. 2007. Absence of CC chemokine ligand 2 does not limit obesity-associated infiltration of macrophages into adipose tissue. Diabetes. 56:2242-2250. 